\title{
Sexuell erregte Frauen ekeln sich weniger
}

\begin{abstract}
Ohne Sex wäre die Menschheit längst ausgestorben. Ohne die Fähigkeit, sich zu ekeln, unter Umständen aber auch, bewahrt uns doch Ekel oft vor dem Kontakt mit möglicherweise krankheitserregenden oder giftigen Stoffen. Nun sind beim Sex Körperflüssigkeiten und -gerüche involviert, die durchaus auch ekelerregend sein können. Wie kommt es dann, so fragten sich niederländische Wissenschaftler, dass sich Menschen trotz des Ekelfaktors immer wieder zu sexuellen Aktivitäten durchringen können?
\end{abstract}

- Die Forscher teilten 90 Frauen mit einem Durchschnittsalter von 23 Jahren in drei gleich große Gruppen auf. 30 Teilnehmerinnen bekamen einen Sexfilm zu sehen, den andere Frauen - in einem Vorversuch - als besonders erregend bewertet hatten. Die zweite Gruppe sah Sportclips, die zwar erregend, aber nicht sexuell anregend sein sollten. Die dritte Gruppe bekam Aufnahmen einer Zugfahrt gezeigt. Die Filme hatten jeweils eine Länge von 35 Minuten. Die Vorführung wurde mehrmals unterbrochen und erst fortgesetzt, nachdem die Probandinnen bestimmte Aufgaben mit mehr oder weniger hohem Ekelpotential absolviert hatten. So sollte beispielsweise gebrauchtes Toilettenpapier aus einem Gefäß herausgeholt und wieder zurückgetan werden (das Papier war in Wirklichkeit mit Schokolade verschmiert), oder es sollte Saft aus einem Glas getrunken werden, in dem scheinbar ein totes Insekt schwamm (in Wirklichkeit eine Plastiknachbildung).

Insgesamt waren 16 derartige Aufgaben zu absolvieren. Die Probandinnen mussten anschließend notieren, wie der gezeigte Film auf sie gewirkt hatte und als wie ekelig sie die gestellten Aufgaben empfanden.

Die Gruppe erregter Frauen bewertete vor allem die Ekelaufgaben, die irgendwas mit Sex zu tun hatten, als weniger abstoßend als die anderen Teilnehmerinnen. Auf einer Skala von Null bis Zehn bewerteten sie die Tests mit einem Durchschnittswert von 5,4 - die Probandinnen der anderen Gruppe stuften sie mit 6,9 und 6,6 als ekliger ein. Sie erledigten auch die meisten der Aufgaben (89\%), während die Sportclips-Gruppe im Schnitt nur 65\% und die neutrale 74\% der Tests schafften. Die Teilnehmerinnen, die einen "frauenfreundlichen Softporno" gesehen hatten, stuften sich selbst als weniger geekelt ein und erledigten mehr abstoßende Aufgaben als andere. Die Frauen, die nur einen Sportfilm oder einen neutralen Film gesehen hatten, waren den Wissenschaftlern zufolge schneller und häufiger angeekelt.

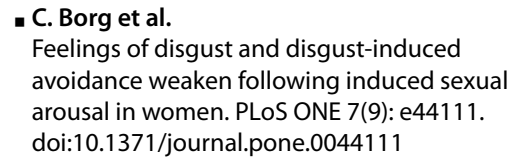

\section{Kommentar}

Sex mag schmutzig sein, aber die meisten Menschen scheint das nicht zu stören, schreiben die Autoren in der Studie. Und das, obwohl erotische Mitbringsel wie Speichel, Schweiß, Sperma und Körpergeruch zu den stärksten Ekelauslösern gehören. Für die Fortpflanzung ist es daher wichtig, dass die sexuelle Erregung die Ekelschwelle anhebt. Frühere Versuche haben übrigens gezeigt, dass der Effekt auch bei Männern im sexuell erregten Zustand auftritt.

K. MALBERG =

\section{Die Glasform bestimmt den Alkoholkonsum}

\begin{abstract}
Wie schnell und wie viel Alkohol getrunken wird, hängt offenbar auch von der Form des Trinkgefäßes ab. Darauf deutet eine Studie britischer Wissenschaftler hin. Aus geraden Bierkrügen wird viel langsamer getrunken als aus geschwungenen.
\end{abstract}

- 159 Testtrinker wurden dazu angehalten, alkoholische Getränke oder Softdrinks aus verschieden geformten Gläsern zu konsumieren. Dabei zeigte sich, dass Bierkrüge mit geraden Wänden um $60 \%$ langsamer geleert wurden als solche mit geschwungenen Wänden (bei gleicher Ausgangsmenge). Das galt für alkoholische, nicht aber für nicht alkoholische Getränke.

\section{Kommentar}

Trinker, so vermuten die Forscher, haben zumindest unbewusst ein Gefühl für eine "angemessene" Trinkgeschwindigkeit. Bei Gläsern mit geschwungenen Wänden werden die Trinkmenge und die Trinkgeschwindigkeit falsch eingeschätzt, wodurch die geschwungenen Gläser zu einem höheren Alkoholkonsum verleiten, als eigentlich geplant.

K. MALBERG -

- A. T. Attwood et al.

(University of Bristol, School of Experimental Psychology, 12a Priory Road, Clifton, Bristol, B88, 15 TU, England, UK; E-mail: angela.attwood@bristol.ac.uk) Glas shape influences consumption rate for alcoholic beverages. PLoS ONE 7(8): e43007. doi:10.1371/journal. pone. 0043007 